Bull. Mater. Sci., Vol. 21, No. 3, June 1998, pp. 227-231. (C) Indian Academy of Sciences.

\title{
Optical and mechanical characteristics of zinc sulphide-thorium fluoride mixed composition thin films for use in the near infrared region $(1-10 \mu \mathrm{m})$
}

\author{
A BASU, B S VERMA, T K BHATTACHARYYA, M KAR and R BHATTACHARYYA* \\ Thin Film and Amorphous Materials Group, National Physical Laboratory, New Delhi 110012 , India
}

MS received 25 November 1997

\begin{abstract}
Mixed composition thin films of zinc sulphide-thorium fluoride have been deposited on glass and silicon substrates by thermal evaporation of mixtures of these materials in different proportions, from a single resistively heated source. The films are characterized for their optical properties (refractive index and extinction coefficient), mechanical properties (intrinsic stress), surface morphology and chemical composition. It is found that these films have tailorable refractive indices and low losses, and that films with certain compositions have low intrinsic stress and smooth surface morphology, making them suitable for incorporation in thin film multilayers for use in the near infrared region up to at least $10 \mu \mathrm{m}$.
\end{abstract}

Keywords. Mixed composition thin films; zinc sulphide-thorium fluoride; optical and mechanical characteristics; near IR region.

\section{Introduction}

It is well known that there are relatively few single materials available which are suitable for incorporation in thin film multilayer stacks useful in the near infrared region $(1-10 \mu \mathrm{m})$. The fabrication of multilayer optical coatings with specified spectral characteristics in this region would be greatly simplified if low loss optical materials with tailorable refractive indices (especially in the low index range) and acceptable mechanical properties (low intrinsic stress, adequate hardness, etc) are available. Mixed composition films offer the possibility of solving this problem. So far, most studies of mixed composition films have been confined to the visible region. Only in recent years has interest been shown in mixed composition films useful in the near infrared region (Sankur et al 1988, 1991; Targrove and Murphy 1990; Gluck et al 1992, 1993). The mixed composition films reported so far, along with their regions of transparency, are listed in table 1.

Zinc sulphide and thorium fluoride are high and low index materials which are used for fabrication of multilayers in the near IR region. We therefore investigated mixed composition thin films of zinc sulphide and thorium fluoride in the search for a suitable thin film material with desirable properties.

\section{Deposition of thin films of $\mathrm{ZnS}^{-\mathrm{ThF}_{4}}$}

Both zinc sulphide and thorium fluoride have the same

*Author for correspondence evaporation temperature $\left(1100^{\circ} \mathrm{C}\right)$. Therefore, mixtures of the two materials could be evaporated from the same resistively heated source. The two materials were taken in different proportions by weight (measured by a Mettler microbalance), ground and mixed together thoroughly in a mortar and pestle and put into a tungsten boat, covered with a perforated shield to prevent spattering of the material. The thermal evaporation was carried out under a vacuum of about $3 \times 10^{-6} \mathrm{mbar}$, and the thickness of the deposited film was monitored by an optical thickness

Table 1. Mixed composition films reported in the literature.

\begin{tabular}{|c|c|c|}
\hline $\begin{array}{l}\text { Composition } \\
\text { of film }\end{array}$ & $\begin{array}{c}\text { Region of } \\
\text { transparency }(\mu \mathrm{m})\end{array}$ & Reference \\
\hline $\mathrm{ZnSe}-\mathrm{LaF}_{3}$ & $0 \cdot 8-2 \cdot 0$ & Gluck et al $(1992,1993)$ \\
\hline $\mathrm{ZnSe}-\mathrm{SrF}_{2}$ & $0 \cdot 6-8 \cdot 0$ & $\begin{array}{l}\text { Sankur et al (1991), } \\
\text { Gluck et al (1993) }\end{array}$ \\
\hline $\mathrm{Si}-\mathrm{YF}_{3}$ & $1 \cdot 5-7 \cdot 0$ & $\begin{array}{l}\text { Sankur et al (1991), } \\
\text { Gluck et al (1993) }\end{array}$ \\
\hline $\mathrm{Si}-\mathrm{ZnSe}$ & $1.5-7.0$ & Gluck et al $(1992,1993)$ \\
\hline $\mathrm{Si}-\mathrm{ZnS}$ & $1.5-7.0$ & Gluck et al (1993) \\
\hline $\mathrm{LaF}_{3}-\mathrm{BaF}_{2}$ & $0 \cdot 25-2 \cdot 0$ & $\begin{array}{l}\text { Targrove and Murphy } \\
\text { (1990) }\end{array}$ \\
\hline $\mathrm{Ge}-\mathrm{MgF}_{2}$ & $2 \cdot 0-4 \cdot 0$ & Sankur et al (1988) \\
\hline $\mathrm{Ge}-\mathrm{CeF}_{3}$ & $2 \cdot 0-5 \cdot 0$ & Sankur et al (1988) \\
\hline $\mathrm{Ge}-\mathrm{ZnS}$ & $2 \cdot 0-12 \cdot 0$ & Sankur et al (1988) \\
\hline Ge-CdTe & $2 \cdot 0-10 \cdot 0$ & Sankur et al (1988) \\
\hline
\end{tabular}


monitor to keep track of the film thickness. Films of physical thicknesses in the range $0.3-1.0 \mu \mathrm{m}$ were deposited on substrates of ophthalmic glass (for refractive index, extinction coefficient and thickness measurements, as also for SEM and XRD studies), thin glass microslides (for stress measurements) and polished Si wafer pieces (for IR transmittance measurements and XPS studies for the chemical composition).

\section{Determination of refractive index, extinction coefficient and thickness of the films}

The refractive index, extinction coefficient and thickness of the films were determined from the trace of the transmittance vs wavelength of the films by the well known procedure described by Swanepoel (1983). The transmittance of the ophthalmic glass substrate coated with the film was measured as a function of wavelength from about $400-1500 \mathrm{~nm}$. Depending on the thickness of the film, a number of maxima and minima were observed in the spectrophotometric trace. Figures $1 \mathrm{a}$ and b show typical traces for a pure $\mathrm{ZnS}$ film and a mixed composition $\mathrm{ZnS}-\mathrm{ThF}_{4}$ film deposited on glass substrates.
The envelopes of the maxima and minima in these curves were drawn, and the values of transmittance (from actual transmittance curve or from envelope curve) at the wavelengths corresponding to each maximum or minimum were noted. A computer programme was developed which calculated the refractive index, $n$, extinction coefficient, $k$ and film thickness, $d$ at each of these wavelengths, on the basis of these transmittance values. These calculated results are also shown in figures $1 \mathrm{a}$ and $\mathrm{b}$, along with the transmittance curves. The variation in the calculated values of $d$ at the different wavelengths, and the corresponding variations in the refractive indices calculated using these values of $d$, gave estimates of the uncertainties in the values of $n$ and $d$ at each wavelength. The values of $k$ are of the order of $10^{-3}$, which shows that these films are almost transparent in the near infrared region.

The refractive indices, extinction coefficients and thicknesses of a few selected films were also determined from ellipsometric measurements at $546.1 \mathrm{~nm}$ wavelength, and were found to agree with the values determined by the above method to within a few per cent. The thicknesses of a few films were also determined directly by
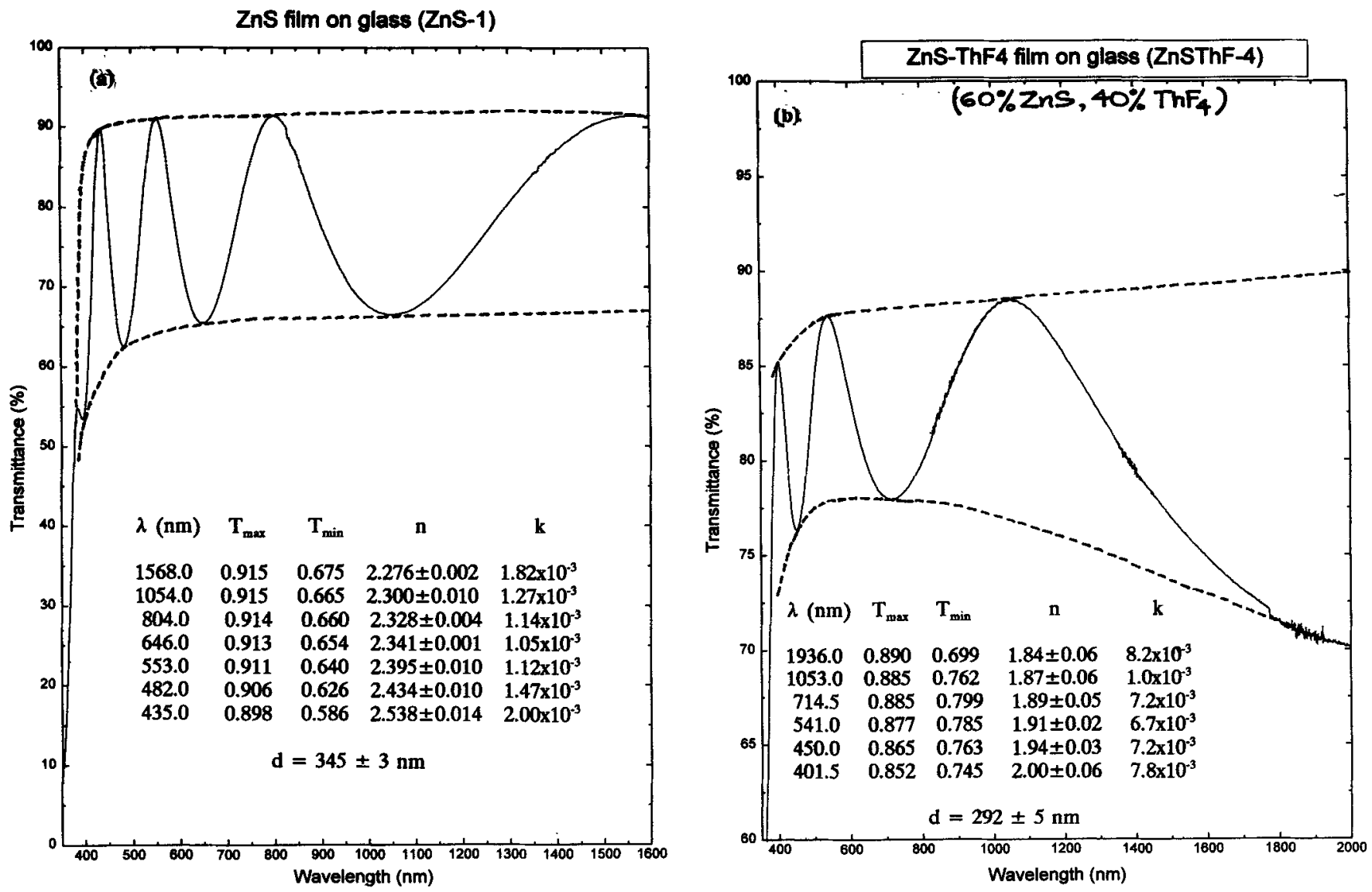

Figure 1. Plots of the transmittance vs wavelength for two films: $\mathrm{ZnS}-1$ (a) and $\mathrm{ZnSThF-4} \mathrm{(b),} \mathrm{deposited} \mathrm{on} \mathrm{ophthalmic} \mathrm{glass}$ substrates. The envelopes of the transmittance maxima and minima are shown. The results of calculations ( $\$ 3$ )-the values of $n$ and $k$ of the film at each of the transmittance extrema, as well as the film thickness-are also shown. 
a stylus scan measurement in a Talystep instrument, and the values were again found to be in good agreement with the previously determined values.

In figure 2 , we have plotted the dispersion curves for the refractive indices of a pure $\mathrm{ZnS}$ film and a film (ZnSThF-4) fabricated from a mixture with $60 \% \mathrm{ZnS}$ and $40 \% \mathrm{ThF}_{4}$ by weight. The refractive index shows a regular decrease with increasing wavelength in both cases, as expected.

In figure 3 , we have plotted the refractive index of each film at $1 \mu \mathrm{m}$ (obtained by interpolation from the refractive index vs wavelength data) against the ratio $f$ for the film, defined as

$$
f=w /(1+w), w=w_{\mathrm{L}} / w_{\mathrm{H}},
$$

where $w_{\mathrm{L}}$ is the weight of $\mathrm{ThF}_{4}$ (low index, $n_{\mathrm{L}}$, constituent) in evaporation mixture and $w_{\mathrm{H}}$ the weight of $\mathrm{ZnS}$ (high index, $n_{\mathrm{H}}$, constituent) in evaporation mixture.

As we shall show later $(\S 7)$, the fractional composition by weight of a film is found to agree with the fractional composition by weight of the evaporation mixture.

There are various models (linear and non-linear) that predict the refractive index of a mixed composition film (Niklasson et al 1981), and some researchers, e.g. Chen et al (1996), have tried to fit their refractive index vs fractional composition data for mixed composition films to one or the other model. However, the scatter in the refractive index vs $f$ data in figure 3 does not warrant the fitting to a particular model. Other researchers (Gluck et al 1992) have also expressed the same opinion. We can, however, say that in the present case the refractive index of the $\mathrm{ZnS}-\mathrm{ThF}_{4}$ mixed composition film varies monotonically with composition (represented by the fraction, $f$ ) and follows an approximately linear relation. In fact, the dotted line in figure 3 is the result of the Linear Model (Niklasson et al 1981), according to which the effective refractive index of the mixed composition film is given by

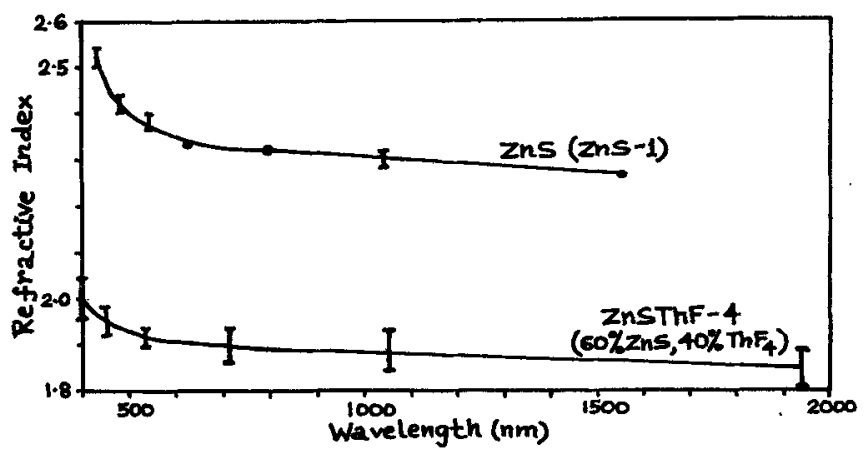

Figure 2. Refractive index (calculated in $\$ 3$ ) vs wavelength for the two films ZnS-1 and ZnSThF-4.

$$
\begin{aligned}
n_{\text {eff }} & =\left(n_{\mathrm{H}} \cdot w_{\mathrm{H}}+n_{\mathrm{L}} \cdot w_{\mathrm{L}}\right) /\left(w_{\mathrm{H}}+w_{\mathrm{L}}\right) \\
& =(1-f) \cdot n_{\mathrm{L}}+f \cdot n_{\mathrm{H}}=n_{\mathrm{L}}+f \cdot\left(n_{\mathrm{H}}-n_{\mathrm{L}}\right),
\end{aligned}
$$

using the expression for $f$ given earlier.

\section{Stress measurement}

The intrinsic stresses in the mixed composition films of $\mathrm{ZnS}-\mathrm{ThF}_{4}$ are expected to be small, since $\mathrm{ZnS}$ films have been reported to have moderate compressive stress and $\mathrm{ThF}_{4}$ films have moderate tensile stress. The intrinsic stresses in the films were determined by a laser scanning set-up. Light from a $\mathrm{HeNe}$ laser was passed through a beam expander and aperture to obtain a collimated beam which would not suffer much divergence over a distance of several metres. The laser beam was split by a beamsplitter into two beams: one was sent off to the side, and the other was directed to the sample under investigation. After reflection from the sample, this beam was directed by the same beamsplitter to a position sensitive quadrant detector, or to a screen a few metres away. The laser beam spot on the sample was translated over the surface of the sample by a micrometer over a distance of several $\mathrm{mm}$, in a direction normal to the incident light, and the resultant deflection of the reflected light beam (which would indicate curvature of the sample surface) was measured by the position sensitive detector or on the screen. For deflections of less than $1 \mathrm{~cm}$, the detector could measure the deflection to an accuracy of $0.1 \mathrm{~mm}$. But greater accuracy was usually obtained by measuring a deflection of a few $\mathrm{cm}$ on the screen.

The intrinsic stress in the film was determined by the following relation:

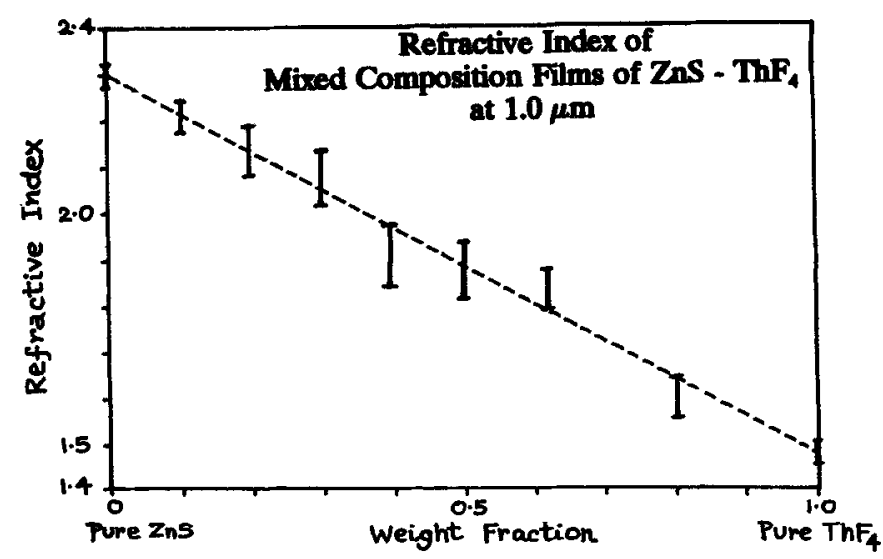

Figure 3. Refractive index at $1 \mu \mathrm{m}$ (calculated in $\S 3$ and interpolated to $1 \mu \mathrm{m}$ ) vs the weight fraction, for various mixed composition films. The dashed line is the plot corresponding to the theoretical Linear Model. 
Film stress $\sigma=\frac{E t_{\mathrm{s}}^{2}}{6 t_{\mathrm{f}}(1-\mu)}\left[\frac{1}{R}-\frac{1}{R_{0}}\right]$

where $E$ is the Young's modulus, $\mu$ the Poisson's ratio, $t_{\mathrm{s}}$ the thickness of substrate, $t_{\mathrm{f}}$ the film thickness and $R$ and $R_{0}$ are the radii of curvature of substrate before and after deposition of film, and

$$
R=2 x L / d,
$$

where $x$ is the displacement of sample, $L$ the distance of screen/detector from sample and $d$ the displacement of beam spot, and similarly for $R_{0}$.

For the thin glass microslide used as the substrate for stress measurements (Corning 0211 alkali zinc borosilicate sheet glass), we used the values [Corning catalog]: $E=7.45 \times 10^{10} \mathrm{~N} / \mathrm{m}^{2}, \mu=0.22, t_{\mathrm{s}}=0.15 \mathrm{~mm}$.

The radius of curvature of the substrate was measured at the centre of the substrate before and after deposition of the film. The results of measurement are shown in figure 4. Compressive stresses are shown as positive and tensile stresses as negative. For ease of representation

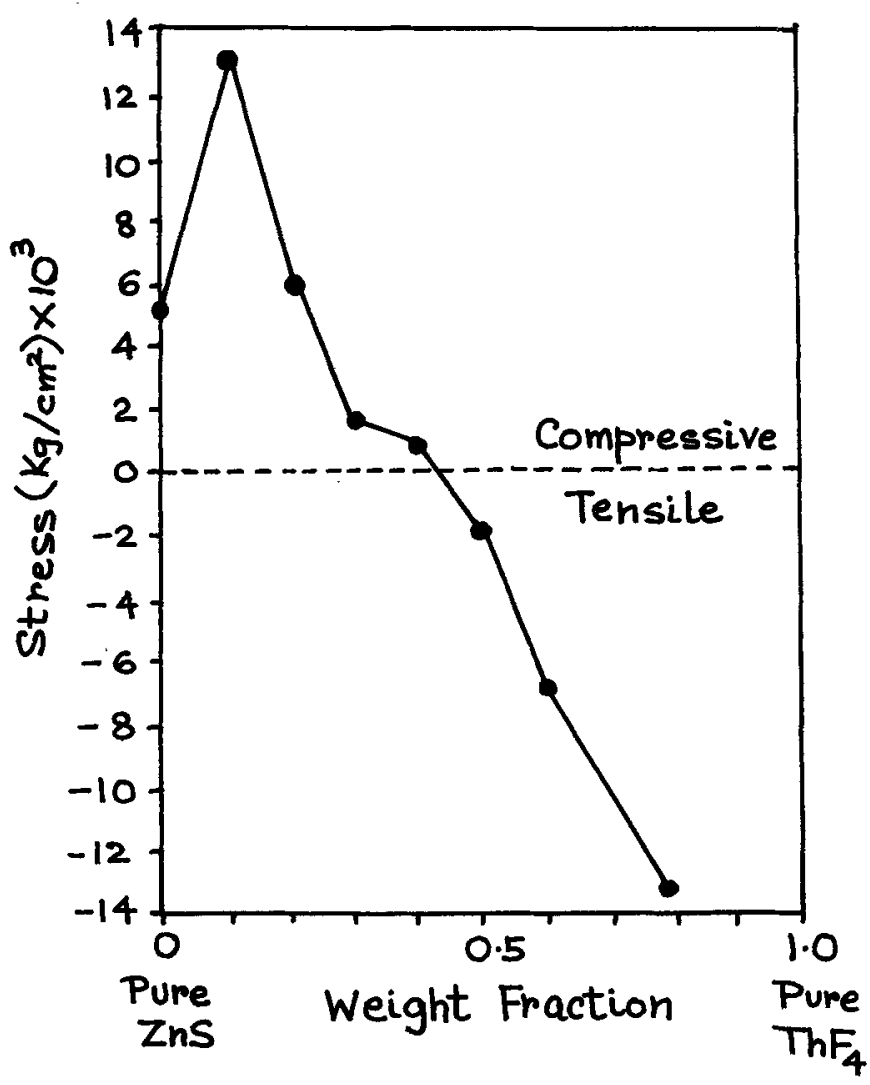

Figure 4. Intrinsic stress (in $\mathrm{kg} / \mathrm{cm}^{2}$ ) vs the weight fraction for the various mixed composition films studied in $\S 4$. Compressive stresses are shown as positive and tensile stresses as negative. of positive and negative stresses in the same figure, and since stresses have usually been expressed in $\mathrm{kg} / \mathrm{cm}^{2}$ in the literature, we have expressed the stresses in our films in $\mathrm{kg} / \mathrm{cm}^{2}$, using the conversion $1 \mathrm{~kg} / \mathrm{cm}^{2}=9.8 \times 10^{4}$ $\mathrm{N} / \mathrm{m}^{2}$. We see that as the weight fraction of $\mathrm{ThF}_{4}$ in the mixed composition films increases, the intrinsic stress changes from compressive to tensile, and a film with a weight fraction of about 0.5 , i.e. a film with about $50: 50$ ratio of $\mathrm{ZnS}-\mathrm{ThF}_{4}$, has very low stress. This is an illustration of the reduction of intrinsic stresses in mixed composition films reported by several researchers (Austin 1973; Sankur et al 1988, 1991; Targrove and Murphy 1990; Gluck et al 1992, 1993).

\section{Surface morphology}

The mixed composition films were examined under a scanning electron microscope to see the surface roughness, cracks or other surface features of the films. The films were examined both at normal incidence and at oblique incidence up to a magnification of $15,000 \times$. However, all the films exhibited featureless surface morphology up to this magnification. We therefore anticipate that these films will exhibit very low optical scattering losses and hence are suitable for use in optical multilayer stacks without introducing appreciable scattering losses.

\section{Transparency in near IR $(1-10 \mu \mathrm{m})$ range}

The IR transmittance spectrum of thin films coated on silicon substrates was measured on a Perkin-Elmer spectrophotometer from $2.5-10 \mu \mathrm{m}$. The spectra showed absorption peaks of the silicon substrate at about $9 \cdot 1 \mu \mathrm{m}$, and were otherwise quite flat over this entire range. We therefore conclude that these thin films are almost transparent over the wavelength range of interest, and are thus suitable for the fabrication of multilayer stacks.

\section{Structure and chemical composition}

$\mathrm{X}$-ray diffraction studies of some of the mixed composition films showed a peak corresponding to zinc sulphide, but there was no defined peak corresponding to thorium fluoride. This is clearly seen in the plots in figure 5 , for films ZnSThF-1 (a), ZnSThF-4 (b) and ZnSThF-8 (c). The films in (a) and (b) have large weight fractions of $\mathrm{ZnS}$, and the diffraction peaks corresponding to $\mathrm{ZnS}$ are clearly seen. The film in (c) is predominantly $\mathrm{ThF}_{4}$, and no diffraction peak is seen here. This indicates that zinc sulphide exists in a crystalline phase in the mixed composition films, while thorium fluoride exists in an amorphous phase.

The chemical composition of the mixed composition films was determined by the XPS technique, where 


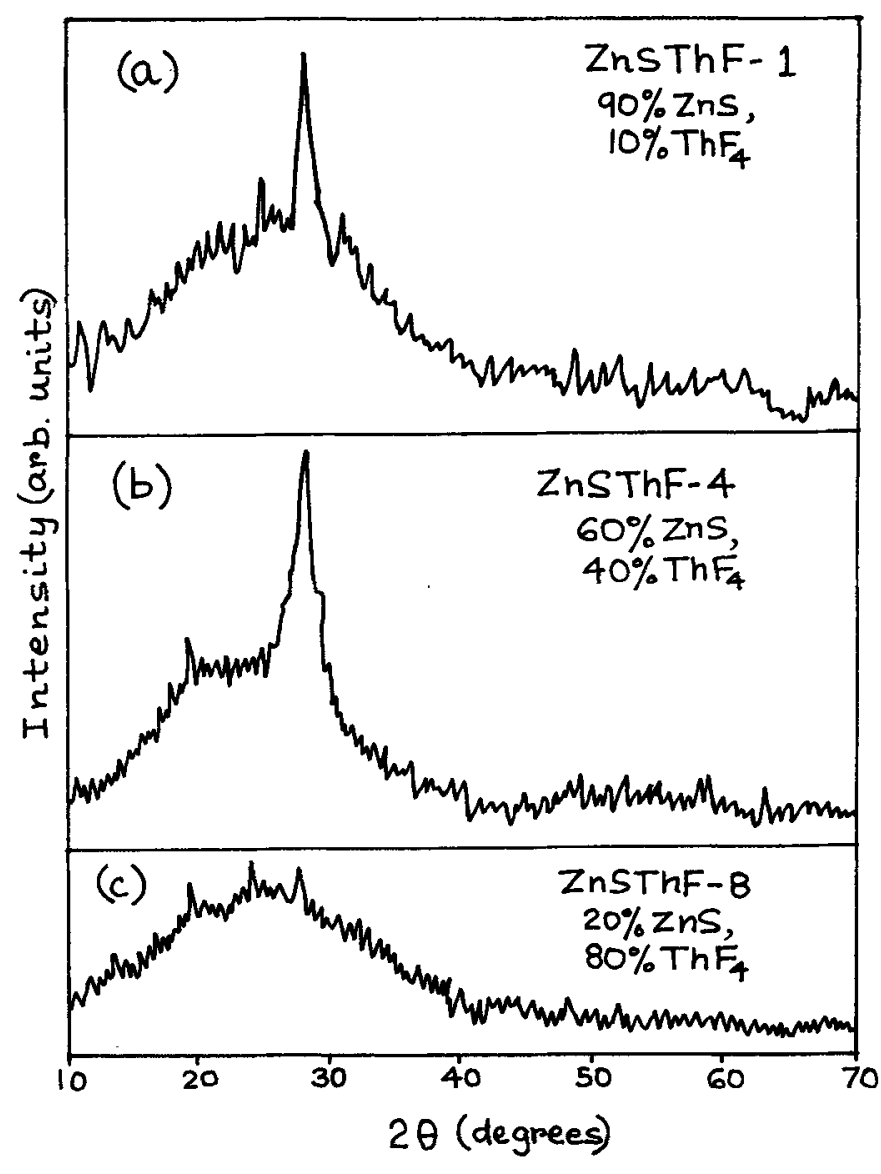

Figure 5. X-ray diffraction plots for three mixed composition films, showing the peak corresponding to $\mathrm{ZnS}$ and the absence of a peak corresponding to $\mathrm{ThF}_{4}$.

surface scans of some of the films showed that the films were comprised of $\mathrm{ZnS}$ and $\mathrm{ThF}_{4}$ only. The weight proportions of $\mathrm{ZnS}$ and $\mathrm{ThF}_{4}$ in the films were found to be quite close to the weight proportions in the evaporation mixture.

\section{Conclusions}

The mixed composition films of zinc sulphide and thorium fluoride have tailorable refractive indices (from about $2.3-1.5$ at $1 \mu \mathrm{m}$ wavelength) and fairly low extinction coefficients, of the order of $10^{-3}$ or lower. The intrinsic stress is very low for film compositions with a $50: 50$ ratio by weight of $\mathrm{ZnS}$ and $\mathrm{ThF}_{4}$. The films exhibit almost featureless surface morphology and therefore should have low optical scattering losses. Further, while $\mathrm{ZnS}$ appears to exist in a crystalline phase in these films, $\mathrm{ThF}_{4}$ appears to exist in an amorphous phase. In conclusion, these mixed composition films should be useful in the fabrication of multilayer stacks to meet specified spectral characteristics in the near IR region.

\section{Acknowledgements}

This work was carried out under a research grant from the Ministry of Science and Technology (Science and Engineering Research Council), Govt of India, for which we are most grateful.

We wish to place on record our gratitude to the several people who helped us to characterize the thin film samples: Mr Sushil Kumar for the stress setup and measurements, Dr S P Verma and Dr D Gupta for the IR transmittance measurements, Dr R P Pant for the $\mathrm{X}$-ray diffraction analysis, $\mathrm{Dr} \mathrm{C}$ Anandan for XPS studies and Mr K B Ravat for the SEM studies.

\section{References}

Austin R R 1973 Appl. Opt. 12665

Catalog for Speciality Glasses and Glass Ceramic Materials, Coming Glass Works, Corning, NY, USA

Chen J-S, Chao S, Kao J-S, Niu H and Chen C-H 1996 Appl. Opt. 3590

Gluck N S, Taber D B, Heuer J P, Hall R L and Gunning W J 1992 Appl. Opt. 316127

Gluck N S, Sankur H O and Taber D 1993 Society of vacuum coaters, 36th annual technical conference proceedings (USA: Society of Vacuum Coaters) p. 119

Niklaason G A, Granquist C G and Hunderi O 1981 Appl. Opt. 2026

Sankur H O, Gunning W J and DeNatale J F 1988 Appl. Opt. 271564

Sankur H O, DeNatale J F and Gunning W J 1991 Appl. Opt. 30495

Swanepoel R 1983 J. Phys. E.: Sci. Instrum. 161214

Targrove J D and Murphy A R 1990 Thin Solid Films 19147 